Journal of Back and

Musculoskeletal Rehabilitation

Journal of Back and Musculoskeletal Rehabilitation 6 (1996) 213

\title{
Announcement \\ Manual Medicine \\ State of the Art 1996
}

June 15th 1996, Münster, Germany

(Under the Patronage of the European Spine Society)

Organisation: M. Schilgen, J. Dvorák, W.H.M. Castro.

Topics: Biomechanics - Neurophysiology - Complications - Imaging Diagnostics - Apparative Functional Analysis - Physical Therapy/Training Therapy - Quality Control - Patient Consent.

Further information

R. Pagenkemper, Akademie für Manuelle Medizin an der Westfälischen Wilhelms - Universität Münster, Von-Esmarch-Str. 56, D-48149 Münster.

Tel. 0251/98130-41; Fax: 0251/98130-39 